\title{
Optimization of Ship Structures Based on Plastic Design
}

\author{
by Hisashi Nobukawa*, Member M. Abdul Rahim**, Member \\ Tetsuya Yao*, Member
}

\begin{abstract}
Summary
In this paper a procedure for the optimization of ship structures is developed based on plastic design of ship structures consisting of the transverse and longitudinal members using the Sequential Unconstrained Minimization Technique (SUMT). Using the developed method, the optimization of a segregated ballast tanker is carried out considering the weight of one hold length as the objective function. Midship section scantlings are chosen as the design variables. Constraints are considered with respect to plastic collapse of hull girder under longitudinal bending, of structural members subjected to axial force, shear force and bending moment, and of platings subjected to lateral pressure. Constraints are also set in buckling strength of platings under inplane load and in design variables such as minimum plate thickness. The variations of maximum bending moments and shear forces due to changes in design variables are calculated based on Taylor series expansion method so as to reduce the computation time of iterative calculation for function minimization in the SUMT procedure. The tanker structure got optimized and the results show that the proposed method based on plastic design is an effective tool in the optimization of ship structures. A comparison of the proposed method with the optimization based on elastic design is also made and the results are presented.
\end{abstract}

\section{Nomenclature}

$a$ : length of the rigid part at end $A$ of a beam $A B$

$A$ : sectional area of a beam

$A_{f}:$ sectional area of a flange

$A_{p}$ : sectional area of a plate

$A_{w}$ : sectional area of a web

$b:$ length of the rigid part at end $B$ of a beam $A B$

$E:$ modulus of elasticity

$G:$ shear modulus

$I$ : moment of inertia

$l:$ length of the non-rigid part of a beam $A B$

$M:$ maximum bending moment

$M_{0}$ : bending moment satisfying fully plastic interaction relationship

$M_{0}^{H}: M_{0}$ for ship hull

$M\left(x_{11}\right)$ : maximum bending moment of the initial ship

$q:$ water pressure acting on a plate

$q_{u}:$ allowable pressure to prevent plastic collapse

$Q$ : shear force

$Q_{0}$ : shear force satisfying fully plastic interaction relationship

$Q_{11}^{H}: Q_{01}$ for ship hull

u': deflection

$x_{w i}: i$ th design variable for the initial ship

* Faculty of Engineering, Hiroshima University

** Graduate School, Hiroshima University

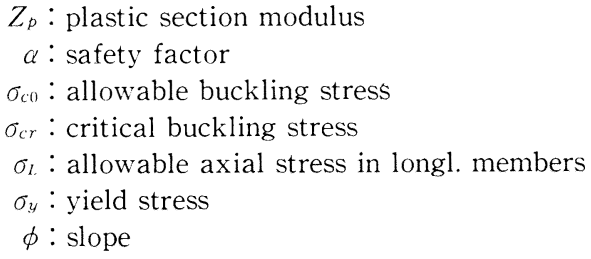

\section{Introduction}

The optimum design of a ship structure is aimed at reducing the hull weight and/or construction cost by optimizing the scantlings and spacings of structural members without sacrificing the functional and strength requirements.

The optimum design of ship structures based on elastic structural analysis had been extensively carried out $^{1-4)}$ using the Sequential Unconstrained Minimization Technique (SUMT), a nonlinear optimization method. However, the optimization based on elastic design results in a margin on the safety factor which leaves further room for optimization, and the concept of plastic design is of help in overcoming this. Recently, sensitivity analysis of elastoplastic structural problems has been investigated ${ }^{5-8)}$ and optimization incorporating elastoplastic sensitivity analysis is of increasing interest. However, in case of ship structures, which are inherently complex, the optimization based on elastoplastic analysis may be difficult and may require more computation time than that based on elastic analysis. 
One method to overcome these problems of massive computation is to initially perform elastic structural analysis to find out the internal forces in transverse (deck, side and bottom frames) and longitudinal (deck and bottom girders) members and then to constrain these with the respective fully plastic conditions in the optimization process.

In the present paper, such a procedure for the optimization of ship structures is developed using the SUMT method incorporating Taylor's approximation with the first and second order sensitivities based on the results of three dimensional structural analysis. Applying the proposed method, the structural optimization of a segregated ballast tanker is carried out. One hold length between two adjacent transverse bulkheads is optimized taking hull weight as the objective function to be minimized with necessary constraints. The wave loads and allowable buckling stresses for the analyses are based on NK Rule. A comparison of the proposed method with the optimization based on elastic design is also made.

\section{Structural Optimization}

\section{1 SUMT Method}

A nonlinear optimization problem can be expressed as

-determine a vector $\boldsymbol{X}$ which

-minimizes $F=f(\boldsymbol{X})$

-subject to $g_{i}(\boldsymbol{X}) \geq 0, \quad i=1,2,3, \cdots, m$.

Here, $\boldsymbol{X}=\left\lfloor x_{1}, x_{2}, \cdots, x_{n}\right\rfloor^{T}$ represents the $n$-dimensional column vector. The vector $\boldsymbol{X}$ consists of design variables $x_{i}$ which may be represented by the dimensions of a structure such as sizes of frames, girders, plate thicknesses, frame spacings, etc.. The function $f(\boldsymbol{X})$ is the objective function to be minimized which, in this paper, is taken as the hull weight. $g_{i}(\boldsymbol{X})$ are the constraints.

In the present paper, the Sequential Unconstrained Minimization Technique (SUMT) is used for the optimization of the objective function. The SUMT method converts a constrained function into an unconstrained function and then finds out its minimum. This is very effective especially when the objective and constraint functions are very complex. Moreover, the optimization algorithm of SUMT is simple and can be executed using computers of relatively smaller size.

In order to minimize the constrained objective function $f(\boldsymbol{X})$, the following unconstrained function $F(\boldsymbol{X}$, $\left.r_{k}\right)$ is defined in the SUMT method.

$$
F\left(\boldsymbol{X}, r_{k}\right)=f(\boldsymbol{X})+r_{k} \sum_{i=1}^{m}\left(\frac{1}{g_{i}(\boldsymbol{X})}\right)
$$

As a starting point of the optimization process, $\boldsymbol{X}=\boldsymbol{X}^{(0)}$ such that $g_{i}\left(\boldsymbol{X}^{(0)}\right)>0$ for all $i$ is initially assumed. Then $r_{1}$ is set such that $F\left(\boldsymbol{X}^{(0)}, r_{1}\right) / f\left(\boldsymbol{X}^{(0)}\right)$ is approximately equal to 1.15-1.2. Proceeding from $\boldsymbol{X}^{(0)}, \boldsymbol{X}=\boldsymbol{X}^{(1)}$ that minimizes $F\left(\boldsymbol{X}, r_{1}\right)$ is determined. Now, starting from $\boldsymbol{X}^{(1)}, \boldsymbol{X}=\boldsymbol{X}^{(2)}$ is determined that minimizes $F\left(\boldsymbol{X}, r_{2}\right)$. Repeating this procedure, $\boldsymbol{X}=\boldsymbol{X}^{(\boldsymbol{k})}(k=1,2,3, \cdots)$ that minimizes $F\left(\boldsymbol{X}, r_{k}\right)$ is calculated. In this iterative calculation, $r_{k}$ is set equal to $r_{k-1} / C 1$ but for $k=1$, with the value of $C 1$ as 10 . The optimum value $\boldsymbol{X}^{(k)}$ can be obtained when $r_{k}$ fully decreases to zero. In the present paper it is assumed that the procedure converges to the optimum when the following condition

$$
|1-| \frac{f\left(\boldsymbol{X}^{(k)}\right)}{f\left(\boldsymbol{X}^{(k-1)}\right)} \mid \leq \varepsilon
$$

is satisfied. In Eq. (2), $f\left(\boldsymbol{X}^{(k)}\right)$ and $f\left(\boldsymbol{X}^{(k-1)}\right)$ are the objective functions in Eq. ( 1 ) minimized at the $k$ th and $(k-1)$ th optimization processes, respectively. In the numerical calculation, $\varepsilon$ is taken as $10^{-6}$. The optimization flow chart is shown in Appendix I.

\section{2 Objective Function and Design Variables}

In the optimum design of ship structures, the objective function can be the hull weight and/or the cost of hull construction. In this paper, the hull weight of one hold length is taken as the objective function. In the evaluation of the objective function, all the structural members are included except lightening holes, collar plates and small tripping brackets.

It is required to reduce the number of design variables to a minimum in order to save the computation time. After careful consideration, nineteen parameters are chosen as the design variables. The first thirteen and the last five of them are shown in Fig. 1 and the fourteenth parameter is the total sectional area of the side shell and the longitudinal bulkheads with attached longitudinal stiffeners. The width of the side ballast tank (web depth of side transverse) is not considered as a design variable because it is a parameter mainly of the volume of the side ballast tank.

\section{3 Constraints}

The constraints considered for the analyses are detailed as follows.

(1) Fully plastic condition of ship hull :

The bending moment and shear force in a hull cross

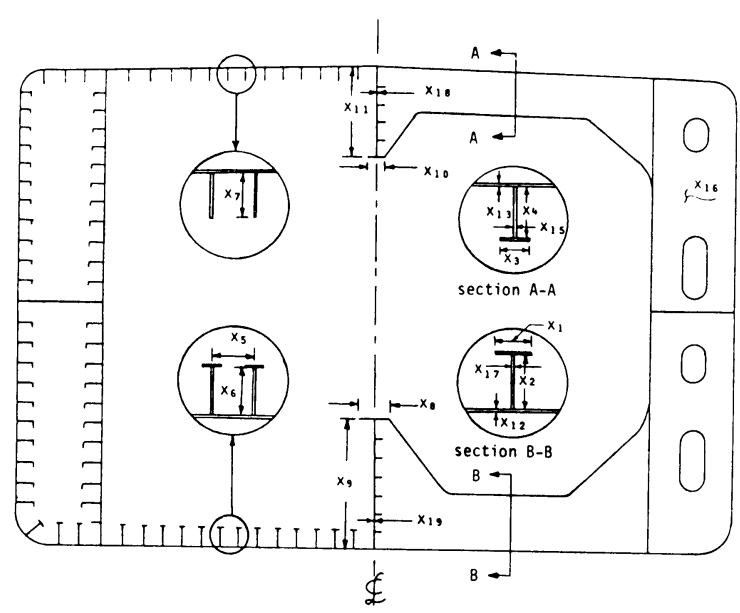

Fig. 1 Midship section of the SBT 
section should not violate the fully plastic condition, subjected to a safety factor.

$$
\begin{aligned}
& g(x)=M_{0}^{\prime \prime}-\alpha_{H} M_{T} \geq 0 \\
& g(x)=Q_{0}^{H}-\alpha_{H} Q_{T} \geq 0
\end{aligned}
$$

where $M_{T}$ and $Q_{T}$ are the maximum bending moment and shear force that may act on the ship hull with an exceeding cumulative probability of $10^{-8}$, and is evaluated as the sum of the respective wave and stil. lwater values.

The fully plastic interaction relationship of bending moment to shear force is derived assuming fully plastic stress distribution in the ship hull cross section.

(2) Fully plastic condition of structural members:

The bending moments, shear forces and axial forces in transverses and longitudinal girders should not violate the respective fully plastic conditions, subjected to a safety factor.

$$
\begin{aligned}
& g(x)=M_{0}-\alpha_{r} \cdot M \geq 0 \\
& g(x)=Q_{01}-\alpha_{r} \cdot Q \geq 0
\end{aligned}
$$

The constraint given by Eq. ( 5 ) is considered for the deck, side and bottom transverses as well as for both the primary and secondary longitudinal members of deck and bottom. The constraint given by Eq. ( 6 ) is considered for the deck, side and bottom transverses.

The fully plastic interaction relationships of bending moment to shear force and axial force are derived assuming fully plastic stress distribution in the cross section of the respective structural members.

(3) Plastic collapse strength of panels:

The water pressure acting on local panels should not exceed the collapse pressure to prevent their plastic collapse, subjected to a safety factor.

$$
g(x)=q_{u}-\alpha_{q} q \geq 0
$$

This constraint is considered for panels in bottom shell and deck plate.

The collapse pressure is evaluated considering plastic mechanism under lateral pressure.

(4) Buckling strength :

The stresses in local panels should not exceed the allowable buckling stresses.

$$
g(x)=\sigma_{c r}-\sigma_{c 0} \geq 0
$$

The elastoplastic buckling constraint is considered for the deck and bottom longitudinals, and for panels in bottom shell and deck plate.

To evaluate elastoplastic buckling stress, Johnson's formula is applied with elastic buckling stress and yield stress.

(5) Minimum values of design variables:

In order to effectively optimize the design variables, it is necessary to set limits on them. Here, lower limits $x_{i m i n}$ are set on the design variables $x_{i}$, and these limits are given in percentage of the initial values of the design variables.

$$
G X_{i}=x_{i}-x_{i \min } \geq 0, \quad i=1,2, \cdots, n .
$$

\section{Structural Analysis}

\section{1 Application of Transfer Matrix Method}

Three dimensional structural analysis is carried out

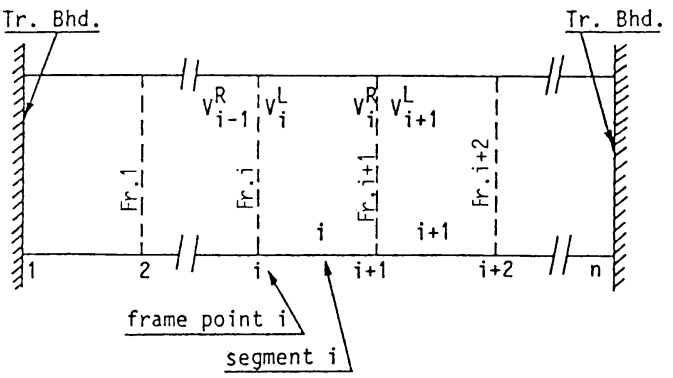

Fig. 2 Mathematical model of one hold length

using the Transfer Matrix Method. The main members considered for the analysis are the deck and bottom members, both transverse and longitudinal. One hold length, the mathematical model of which is shown in Fig. 2, is considered for analysis. The structure has $n$ frames and thus $n-1$ segments, and one segment length corresponds to one frame spacing. The forward and aft ends of the hold bounded by the transverse bulkheads are assumed to be fixed.

The boundary conditions at the aft and forward bulkheads are given by Eqs. (10) and (11), respectively.

$$
\begin{aligned}
& \boldsymbol{V}_{1}^{\boldsymbol{L}}=\boldsymbol{R}_{L} . \boldsymbol{A} \\
& \boldsymbol{R}_{\boldsymbol{R}} \boldsymbol{V}_{n}^{R}=0
\end{aligned}
$$

The transferences of state vectors from left end to right end in a segment $i$ and from left to right at a frame point $i$ are done using Eqs. (12) and (13), respectively.

$$
\begin{aligned}
& \boldsymbol{V}_{i}^{R}=\boldsymbol{F}_{i} \boldsymbol{V}_{i}^{L} \\
& \boldsymbol{V}_{i}^{L}=\boldsymbol{P}_{i} \boldsymbol{V}_{i-1}^{R}
\end{aligned}
$$

From Eqs. (10), (11), (12) and (13), the following relationship is derived.

$$
\boldsymbol{R}_{R} . \boldsymbol{F}_{n} . \boldsymbol{P}_{n} . \boldsymbol{F}_{n-1} \ldots \ldots \ldots \boldsymbol{P}_{3} . \boldsymbol{F}_{2} . \boldsymbol{P}_{2} . \boldsymbol{F}_{1} . \boldsymbol{R}_{L} . \boldsymbol{A}=0
$$

This on simplification yields

$$
\left[\begin{array}{llll}
a_{1,3} & a_{1,4} & a_{1,7} & a_{1,8} \\
a_{2,3} & a_{2,4} & a_{2,7} & a_{2,8} \\
a_{5,3} & a_{5,4} & a_{5,7} & a_{5,8} \\
a_{6,3} & a_{6,4} & a_{6,7} & a_{6,8}
\end{array}\right]\left[\begin{array}{l}
Q_{B} \\
M_{B} \\
Q_{D} \\
M_{D}
\end{array}\right]_{1}^{L}=\left[\begin{array}{l}
-a_{1,9} \\
-a_{2,9} \\
-a_{5,9} \\
-a_{6,9}
\end{array}\right]
$$

Solving Eq. (14), $Q_{B}, M_{B}, Q_{D}$ and $M_{D}$ are derived and thus the initial vector $\boldsymbol{A}$. Now using Eqs. (10), (12) and (13), the state vectors $\boldsymbol{V}_{i}^{L}$ and $\boldsymbol{V}_{i}^{R}$ at each frame point $i$ are obtained, and thus the deflections, slopes, shear forces, bending moments and in turn the stresses at each frame point.

The field matrix $\boldsymbol{F}_{i}$ of the segment $i$ is derived from the slope-deflection equations as well as from the shear force and bending moment equations for the corresponding longitudinal girders. The point matrix $\boldsymbol{P}_{i}$ of the frame point $i$ is derived considering the conditions of continuity of deflections and slopes, and the equilibrium of forces and moments in the longitudinal girders at the frame point $i$.

The state vectors $\boldsymbol{V}_{1}^{L}$ and $\boldsymbol{V}_{n}^{R}$, the initial vector $\boldsymbol{A}$, the end matrices $\boldsymbol{R}_{L}$ and $\boldsymbol{R}_{R}$, the field matrix $\boldsymbol{F}_{i}$ and the 
point matrix $\boldsymbol{P}_{i}$ are given in Appendix II.

\subsection{Application of Taylor Series Expansion Method}

The maximum bending moments and shear forces for the initial ship are calculated by the direct three dimensional analysis mentioned above. However, to save computation time, the subsequent maximum bending moments at each iteration step when design variables are changed are calculated based on Taylor series expansion with sensitivities up to second order using Eq. (15).

$$
\begin{aligned}
M_{\max }= & M\left(x_{0}\right)+\sum_{i=1}^{n} \frac{\partial M}{\partial x_{i}}\left(x_{i}-x_{0 i}\right) \\
& +\frac{1}{2} \sum_{i=1}^{n} \sum_{j=1}^{n} \frac{\partial^{2} M}{\partial x_{i} \partial x_{j}}\left(x_{i}-x_{0 i}\right)\left(x_{j}-x_{0 j}\right)
\end{aligned}
$$

The derivatives in the above equation are numerically evaluated and are given as follows.

$$
\begin{aligned}
& \frac{\partial M\left(x_{1}, x_{2}, \cdots\right)}{\partial x_{1}}=\{ M\left(x_{1}+\Delta x_{1}, x_{2}, \cdots\right) \\
&-\left.M\left(x_{1}-\Delta x_{1}, x_{2}, \cdots\right)\right\} /\left(2 \Delta x_{1}\right) \\
& \frac{\partial^{2} M\left(x_{1}, x_{2}, \cdots\right)}{\partial x_{1} \partial x_{2}}=\left\{M\left(x_{1}+\Delta x_{1}, x_{2}+\Delta x_{2}, \cdots\right)\right. \\
&-M\left(x_{1}+\Delta x_{1}, x_{2}-\Delta x_{2}, \cdots\right) \\
&-M\left(x_{1}-\Delta x_{1}, x_{2}+\Delta x_{2}, \cdots\right) \\
&\left.+M\left(x_{1}-\Delta x_{1}, x_{2}-\Delta x_{2}, \cdots\right)\right\} \\
& /\left(4 \Delta x_{1} \Delta x_{2}\right) \\
& \partial^{2} M\left(x_{1}, x_{2}, \cdots\right)=\left\{M\left(x_{1}+\Delta x_{1}, x_{2}, \cdots\right)\right. \\
& \partial x_{1}^{2}-2 M\left(x_{1}, x_{2}, \cdots\right) \\
&\left.+M\left(x_{1}-\Delta x_{1}, x_{2}, \cdots\right)\right\} /\left(\Delta x_{1}\right)^{2} \\
& \Delta x_{i}=\text { increment in } x_{i}=0.05 x_{i}
\end{aligned}
$$

The subsequent maximum shear forces at each itera. tion step are also similarly calculated.

\section{3 Constraints Due to Fully Plastic Interactions Between Internal Forces}

In order to calculate the fully plastic interaction relationships between internal forces, the members are categorized into two groups as follows:

1) Members subjected to axial force and bending moment

-Deck and bottom center girders

Deck and bottom longitudinals

2 ) Members subjected to shear force and bending moment

\section{- Transverse ring members (deck, side and bottom) \\ Ship hull}

The yield function $\Gamma$ of a beam-bar subjected to axial force and bending moment can be written as

$$
\begin{aligned}
& \Gamma=m-\frac{1}{Z_{p}}\left(a_{1}+b_{1} n+c_{1} n^{2}\right)=0 \\
& \text { for }-1 \leq n<-1+\frac{2 A_{f}}{A} \\
& \Gamma=m-\frac{1}{Z_{p}}\left(a_{2}+b_{2} n+c_{2} n^{2}\right)=0 \\
& \text { for }-1+\frac{2 A_{f}}{A} \leq n<1-\frac{2 A_{p}}{A}
\end{aligned}
$$

$$
\begin{aligned}
& \Gamma=m-\frac{1}{Z_{p}}\left(a_{3}+b_{3} n+c_{3} n^{2}\right)=0 \\
& \qquad \text { for }-1-\frac{2 A_{p} \leq n \leq 1}{A} \leq
\end{aligned}
$$

where

$$
A=A_{f}+A_{w}+A_{p}
$$

$m=M / M_{p}$ is the ratio of the bending moment to the fully plastic bending moment, and $n=I^{\prime} / I_{p}^{\prime}$ is the ratio of the axial force to the fully plastic axial force. $a_{1}, b_{1}$. $c_{1}, a_{2}, b_{2}, c_{2}, a_{3}, b_{3}$ and $c_{3}$ are functions of the scantlings of a member.

The yield function $\Gamma$ of a beam subjected to shear force and bending moment can be written as

$$
\begin{aligned}
\Gamma=m-\frac{1}{Z_{p}}\left(a_{4}+b_{4} v+c_{4} v^{2}\right)=0 & \\
\text { for } & A_{f} \geq A_{p}+A_{u} \\
& \text { or } \\
& A_{f} \geq A_{p} \text { and }|f| \geq F V \\
I=m-\frac{1}{Z_{p}}\left(a_{5}+b_{5} / v+c_{5} v\right)=0 & \text { for }|f| \leq F l \\
\Gamma=m-\frac{1}{Z_{p}}\left(a_{6}+b_{6} v^{2}+c_{6} v^{2}\right)=0 & \\
\text { for } & A_{p} \geq A_{f}+A_{u} \\
& \text { or } \\
& A_{f} \leq A_{p} \text { and }|f| \geq F l
\end{aligned}
$$

where

$$
\begin{aligned}
& F V=\sqrt{ } 1-\left[\left(A_{p}-A_{i}\right) / A_{u}\right]^{2} \\
& v^{\prime}=\sqrt{ } 1-f^{2}
\end{aligned}
$$

and $f=F / F_{p}$ is the ratio of the shear force to the fully plastic shear force. $a_{4}, b_{4}, c_{4}, a_{5}, b_{5}, c_{5}, a_{6}, b_{6}$ and $c_{6}$ are functions of the scantlings of a member.

The typical fully plastic interaction relationships represented by Eqs. (16) and (17) are plotted in Fig. 3 ( a ) and (b), respectively. From the structural analysis for the initial ship, the ratios $n / m$ and $f / m$ are evaluated as

$$
\begin{aligned}
& n / m=C_{n} \\
& f / m=C .
\end{aligned}
$$

As the intersecting point of Eqs. (16) and (18), $m_{m}$ is obtained satisfying the fully plastic interaction relationship, and in turn the corresponding maximum bending moment $M_{0}$ as follows.

$$
M_{0}=m_{0} M_{p}
$$

From this, the bending moment constraint due to fully plastic condition is calculated using Eq. (5). In the same manner, the maximum bending moment and shear force for constraints based on Eqs. (17) and (19) are derived.

\section{Optimization of a Segregated Ballast Tanker}

\section{1 Calculation Procedure}

Using the method detailed above, the optimization of one hold structure of a segregated ballast tanker (SBT is carried out. The main particulars of the SBT are Length B. P. $=224.00 \mathrm{~m}$ : Breadth $($ mld. $)=32.20 \mathrm{~m}$ 


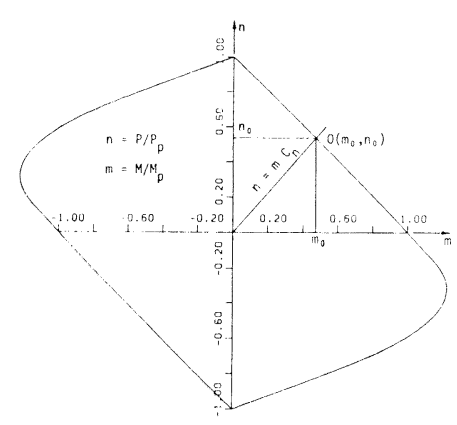

(a) Longitudinal members

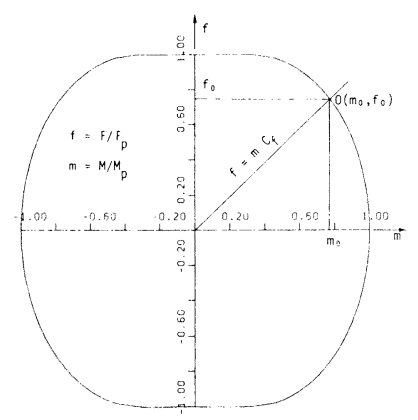

(b) Transverse members

Fig. 3 Fully plastic interaction curves

Depth $(\mathrm{mld})=.19.40 \mathrm{~m}:$ Draught $\quad=12.17 \mathrm{~m}$

Dead Weight $=61,000 \mathrm{t}:$ Block Coeft. $=0.832$

One hold length bounded by two transverse bulkheads is taken for optimization, considering the hull weight as the objective function. The hold configuration is shown in Fig. 4, and the midship section in Fig. 1. For analysis, the structural members in the midship section are simplified to beam-bars with rigid portion at both ends as shown in Fig. 5. The length of the rigid portion is evaluated based on the concept of span point ${ }^{4)}$. The

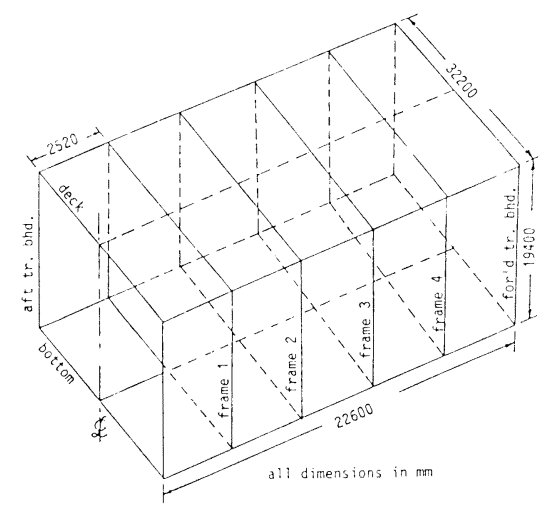

Fig. 4 configuration of one hold length

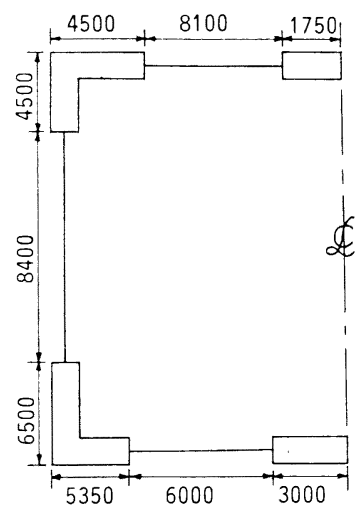

Fig. 5 Idealized midship section

optimization is carried out for ten cases ( $A 1$ to $A 10$ ) by changing $r_{1}$ from $10^{3}$ to $10^{6}$ and $\boldsymbol{X}_{\min }$ from $90 \%$ to $50 \%$ of $x_{i}$, one at a time, keeping other parameters constant. The basic parameters of the various cases are shown in Table 1.

The optimization is carried out based on wave loads presented in NK Rule ${ }^{1(1)}$. Both ballast and full load conditions are considered, which are indicated in Fig. 6. The allowable stresses based on NK Rule are shown in Table 2. The safety factor for both transverse and longitudinal members is taken as 1.5 and that for hull section is taken as 1 . The bending moment, shear force and bending stress distributions are calculated for the initial ship in both ballast and full load conditions. The maximum value of the bending moments in the respective members are used in the analyses. The sensitivities of the maximum bending moments with respect to the design variables are required to effectively optimize the structure with minimum computation cost, and are calculated for the initial ship. The value of $m_{0}$ in Eq. (20) for various structural members are shown in Table 3 for both ballast and full load conditions.

\section{2 Optimization Results}

\section{2. 1 Standard Ship}

In the case of the standard ship, the number of frame spaces $(N)$ is five in a hold length and the optimization is carried out with $N=5$. Since the effect of shear force is small, the constraint given by Eq. ( 6 ) is not consid-

Table 1. Basic parameters of various cases

\begin{tabular}{|l|c|c|c|c|c|c|}
\hline Cases & $N$ & $X_{\text {m } 1 \mathrm{n}}$ & $\mathrm{r}_{1}$ & $\begin{array}{c}\text { Tr.Fr.Sp. } \\
\text { (min) }\end{array}$ & $\begin{array}{c}\text { Iteration } \\
\text { number }\end{array}$ & $\begin{array}{c}\text { Height } \\
\text { (tons) }\end{array}$ \\
\hline $\mathrm{A} 1$ & 5 & $90 \%$ & $5.0 \mathrm{E} 4$ & 4520.0 & 26912 & 919.42 \\
$\mathrm{~A} 2$ & 5 & $80 \%$ & $5.0 \mathrm{E} 4$ & 4520.0 & 17951 & 860.28 \\
$\mathrm{~A} 3$ & 5 & $70 \%$ & $5.0 \mathrm{E} 4$ & 4520.0 & 19369 & 781.74 \\
$\mathrm{~A} 4$ & 5 & $60 \%$ & $5.0 \mathrm{E} 4$ & 4520.0 & 16313 & 734.05 \\
$\mathrm{~A} 5$ & 5 & $50 \%$ & $5.0 \mathrm{E} 4$ & 4520.0 & 14301 & 719.23 \\
& & & & & & \\
$\mathrm{~A} 6$ & 5 & $70 \%$ & $1.0 \mathrm{E} 3$ & 4520.0 & 15233 & 785.74 \\
$\mathrm{~A} 7$ & 5 & $70 \%$ & $1.0 \mathrm{E} 4$ & 4520.0 & 13423 & 784.16 \\
$\mathrm{~A} 8$ & 5 & $70 \%$ & $5.0 \mathrm{E} 4$ & 4520.0 & 19369 & 781.74 \\
$\mathrm{~A} 9$ & 5 & $70 \%$ & $1.0 \mathrm{E} 5$ & 4520.0 & 17347 & 804.39 \\
$\mathrm{~A} 10$ & 5 & $70 \%$ & $1.0 \mathrm{E} 6$ & 4520.0 & 15533 & 807.89 \\
\hline
\end{tabular}


Table 2. Allowable stresses (Plastic design)

\begin{tabular}{|c|c|}
\hline Notation & $\begin{array}{c}\text { Stress } \\
\left(\mathrm{kgf} / \mathrm{mm}^{2}\right)\end{array}$ \\
\hline$\sigma_{\mathrm{L}}$ & 18.0 \\
$\sigma_{\mathrm{y}}$ & 28.0 \\
$\sigma_{\mathrm{co}}$ & 20.0 \\
\hline
\end{tabular}
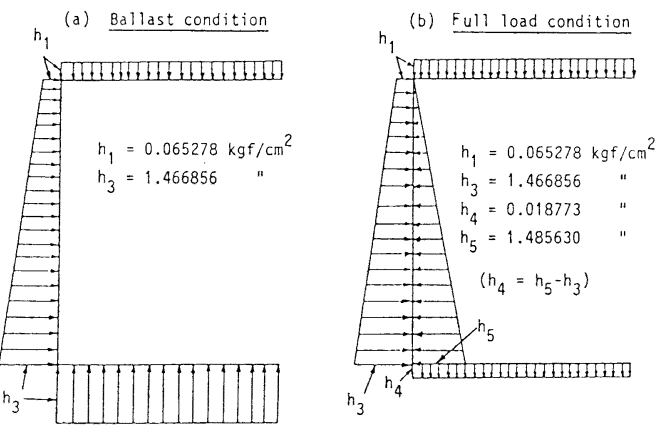

Fig. 6 Loading on the ship hull

Table 3. Values of $m_{0}$ for structural members

\begin{tabular}{|l|c|c|}
\hline \multirow{2}{*}{$\begin{array}{c}\text { Structural } \\
\text { Member }\end{array}$} & \multicolumn{2}{|c|}{$\mathrm{m}_{\mathrm{o}}$} \\
\cline { 2 - 3 } & Ballast & Full load \\
\hline Deck Trans. & 0.8365 & 0.8121 \\
Side Trans. & 0.9738 & 0.9738 \\
Bottom Trans. & 0.8575 & 0.8575 \\
Deck Lgl Gdr. & 0.1217 & 0.1419 \\
Btm. Lgl Gdr. & 0.3011 & 0.1005 \\
Deck Lgl. & 0.1324 & 0.1324 \\
Bottom Lgl. & 0.2894 & 0.0834 \\
Hull Section & 0.9453 & 0.9453 \\
\hline
\end{tabular}

ered in this case and hence the design parameters $x_{15}$ to $x_{19}$ are also not considered as design variables. The maximum bending moment in a hull cross section $\left(M_{T}\right)$ is calculated based on ${ }^{11}$.

In cases $A 1$ to $A 5, \boldsymbol{X}_{\min }$ is varied from $90 \%$ to $50 \%$ in order to check the accuracy of the Taylor's approximation used in the bending moment calculation by evaluating the change in moments in primary structural members. It is defined as

$$
\% \text { change in moment }=\frac{\left(M_{\mathrm{dir}}-M_{\mathrm{apr}}\right)}{M_{\mathrm{dir}}} \times 100
$$

where $M_{\mathrm{dir}}$ and $M_{\mathrm{apr}}$ are the maximum bending moments obtained by direct calculation and by using Taylor's approximation respectively for the optimized ship.

It has been found from Table 4 that the percentage change in moment increases as $\boldsymbol{X}_{\min }$ decreases. The allowable change is set as $10 \%$ which is assumed to provide sufficient accuracy. This necessitated lower limits in the design variables which are incorporated as constraints $G X 1$ to $G X 14$. To make the optimization effective, the lower limit has to be a minimum within the allowable change in moment of $10 \%$. Thus case $A 3$
Table 4. Percentage change in moments for $\mathrm{N}=5$

\begin{tabular}{|c|c|c|c|c|c|c|c|}
\hline \multirow{2}{*}{$\begin{array}{l}\text { CIINAGE IN } \\
\text { MOMFNTS IN } x\end{array}$} & \multirow{2}{*}{$\begin{array}{l}\text { Nota- } \\
\text { tion }\end{array}$} & \multirow{2}{*}{$\begin{array}{l}\text { Initial } \\
\text { Values }\end{array}$} & \multicolumn{5}{|c|}{ VALUES FOR CASFS } \\
\hline & & & A1 & $A 2$ & A3 & A4 & $\Lambda 5$ \\
\hline BALLLAST & & & & & & & \\
\hline Deck Trans & CM1 & 0.00 & 0.00 & 0.35 & 1.53 & 3.08 & 2.54 \\
\hline Side Trans & CM2 & 00 & 0.36 & 0.81 & 1.14 & 2.06 & -0.03 \\
\hline Bottom Trans & СM3 & 0.0 & 0.18 & 0.09 & 0.26 & 1.97 & 0.13 \\
\hline Deck I.gl Gr & $\mathrm{CM}_{4}$ & 0.00 & -3.48 & -6.40 & 9.50 & -12.01 & -11.16 \\
\hline Bottom Lgl Gr & CM5 & 0.00 & -0.89 & -1.74 & -2.82 & -7.86 & -0.04 \\
\hline FULL, $1.0 A D$ & & & & & & & \\
\hline Deck Trans & CM6 & 0.0 & -1.25 & -4.61 & 7.21 & -8.93 & -10.17 \\
\hline Side Trans & $\mathrm{Cr}$ & 0 . & 0.01 & 0.01 & 0.02 & 0.01 & 0.07 \\
\hline Bottom Trans & $\mathrm{CM}$ & 0.0 & 0.09 & -0.09 & 0.08 & 1.65 & -0.47 \\
\hline Deck L.gl Gr & CM9 & 0.00 & -1.93 & -4.19 & -6.86 & -9.96 & 13.82 \\
\hline Bottom Lgl Gr & CM10 & 0.00 & 0.78 & -1.53 & -2.46 & -5.33 & .0 .43 \\
\hline
\end{tabular}

Table 5. Optimized values of design variables for $\mathrm{N}=$ 5 (Plastic design)

\begin{tabular}{|c|c|c|c|c|c|c|c|}
\hline \multirow{2}{*}{$\begin{array}{c}\text { DESIGN } \\
\text { VARIABIE.S }\end{array}$} & \multirow{2}{*}{$\begin{array}{l}\text { Notation } \\
\text { (unit) }\end{array}$} & \multirow{2}{*}{$\begin{array}{l}\text { Initial } \\
\text { Values }\end{array}$} & \multicolumn{5}{|c|}{ OPTIMIZFE VALUES FOR CASES } \\
\hline & & & A6 & A7 & $\Lambda 8$ & A9 & A10 \\
\hline $\mathrm{FlW}$ & 10 & & 39.38 & 36.29 & & & 56.38 \\
\hline ab $D$ & & & & & & & \\
\hline D TrFl Wi & 10 & & & & & & \\
\hline 1) 1 & $x_{4} 1 \mathrm{c}$ & 179 & & & & & 125 \\
\hline $\operatorname{l.g}$ & 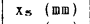 & & & & & & \\
\hline B L & $\left.x_{0}(c)-10\right)$ & & & & & & \\
\hline 1) $1 . \mathrm{gl} \mathrm{Hb}$ & 1 & & & & & & .50 \\
\hline B $|\mathrm{g}|$ Gr F & $x$ & & & & & & 61 \\
\hline B I $\mathrm{gl}$ Gr Wb & & & 10 & & & & 96 \\
\hline $\mathrm{Gr} F$ & & & & & & & \\
\hline D 1 & & & & & & & \\
\hline 11 Thick & & & & & & & \\
\hline & & & & & & & \\
\hline Side Shell Area & $x_{1.4}\left(\mathrm{~cm}^{2}\right)$ & & 13 & 5. & 5. & & \\
\hline & & - & & & & & \\
\hline & & & & & & & \\
\hline & - & & & & & & \\
\hline & W(Tons) & 980.58 & & & 781.74 & & \\
\hline & & & 785.74 & 784.16 & 781.74 & 804.39 & 807.89 \\
\hline
\end{tabular}

is chosen and the corresponding $\boldsymbol{X}_{\min }=70 \%$ is taken as the basis for other calculations. Now with $\boldsymbol{X}_{\min }$ set at $70 \%$, the optimization is carried out by varying $r_{1}$ from $10^{3}$ to $10^{6}$, which corresponds to cases $A 6$ to $A 10$. The optimized variables for these cases are shown in Table 5 , from which the minimum weight is found to be 781.74 tons and it corresponds to case $A 8$. This is the optimum design.

Figure 7 shows the change in hull weight as the optimization progresses for various $r_{1}$ values, the cases being $A 6$ to $A 10$. It goes without saying that there are a number of local minima for a nonlinear function depending on the starting value of $r_{1}$, and this necessitated the different cases by varying $r_{1}$. A few sample distributions of design variables and constraints in moments in ballast condition are shown in Fig. 8(a)(i) against the iteration number, for case $A 8$. The different stages $r_{1}$ to $r_{10}$ are also shown. It is clear from these figures that the calculations need to be done only up to $r_{5}$ for sufficient accuracy. However, the calculations are done up to $r_{10}$ as a check on accuracy.

In order to verify the effectiveness of the present method of optimization, it is necessary to check the effectiveness of the constraints. From Fig. 8(a)-(i), for case $A 8$, the following conclusions can be made.

-for deck transverse $G X 3, G X 4$ and $G X 13$ are effective

for side transverse GX14 is effective 


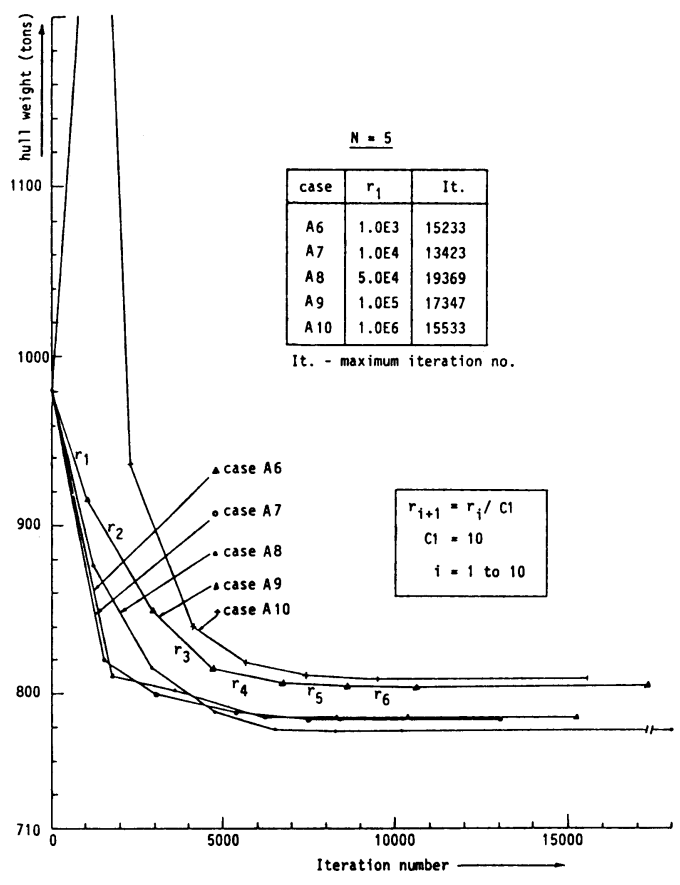

Fig. 7. Hull weight vs. iteration number for various cases for $\mathrm{N}=5$

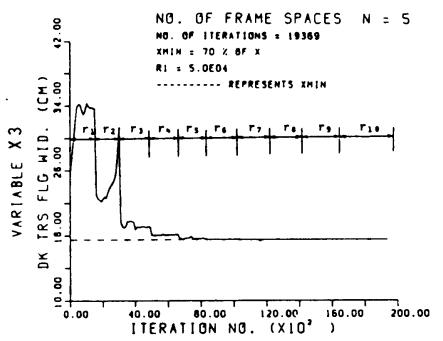

(a)

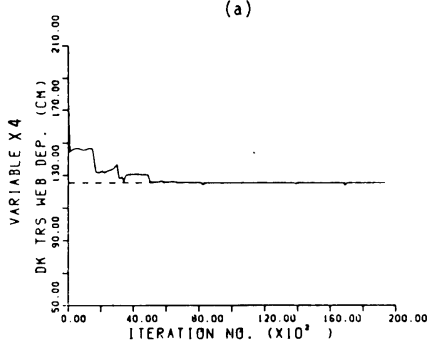

(b)

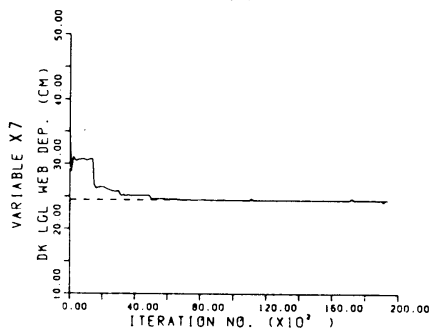

(c)

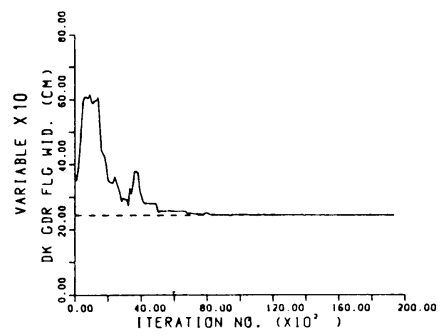

(d)

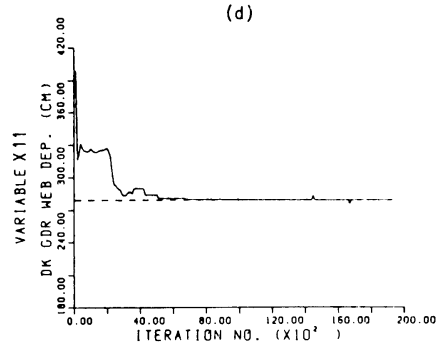

(e)

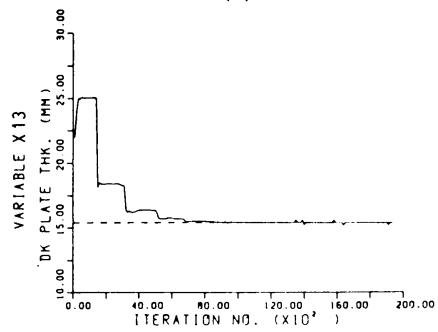

(f)

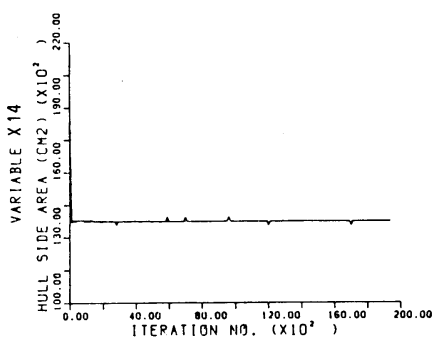

(g)

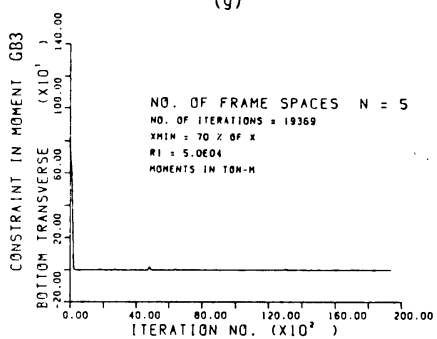

(h)

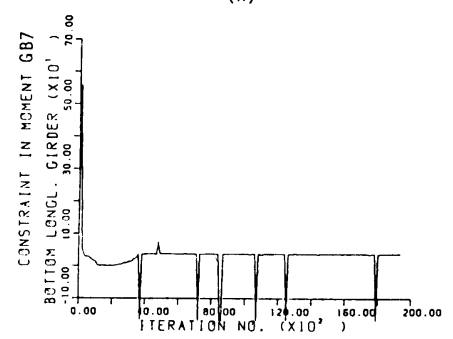

(i)

Fig. 8 Distribution of variable and constraint values for $N=5$ 
transverse is effective. This shows that, depending on the value of $r_{1}$, the constraints that get effective vary. This also necessitates different cases by varying the value of $r_{1}$.

A comparison of the Taylor series expansion method and the direct method was made for case $A 8$. The optimized values of design variables using these methods are almost the same, the hull weights being 781.74 and 780.66 tons for the former and latter cases, respectively. The CPU time taken for the Taylor series expansion method is $1.123 \mathrm{~min}$. against $2.593 \mathrm{~min}$. for the direct method. It may be said that the proposed method using the Taylor series expansion results in a saving of $57 \%$ in CPU time.

4.2.2 Effect of Number of Frame Spaces on Hull Weight

In order to determine the effect of variation in the number of frame spaces $(N)$ on the hull weight, optimization is carried out by varying $N$ from 4 to 7 . It should be noted that the transverse frame spacing varies with variation in $N$ as the hold length is kept as a constant. For each value of $N, r_{1}$ is varied from $10^{3}$ to $10^{6}$ and the design with the minimum weight is chosen as the optimum design, as in the case of the standard ship.

For these cases, the lower limits on the design variables are not considered as $\boldsymbol{X}_{\min }$ cannot be set but for the case of $N=5$ and thus $\boldsymbol{X}_{\min }$ is taken as $0 \%$. Because of this, the Taylor series expansion method cannot be used to evaluate the maximum bending moments and shear forces at each iteration step as the percentage changes in moments may exceed the allowable limit. Hence, direct three dimensional analysis is used to calculate the maximum bending moments and shear forces at each iteration step. The factor of safety is taken as 1.0 for all the structural members.

Based on sample calculations it has been found that the web thicknesses of deck and bottom longitudinal girders ( $x_{18}$ and $x_{19}$, respectively) are not getting reduced and hence they are not considered as design variables for this analysis. The value of $Q_{T}$ in Eq. (4) is assumed to be $\Delta / 10$, where $\Delta$ is the displacement of the ship.

From the optimization results of these cases, it has been found that:

1) Both the constraints given by Eqs. ( 3 ) and (4) are effective for the hull girder.

2 ) The constraint given by Eq. ( 5 ) is effective for the bottom transverse and the deck longitudinal girder.

3 ) The constraint given by Eq. (6) is effective for the side transverse.

Since transverses, longitudinals and girders interact each other, as detailed in Ch. 4.2.1, the assumed constraints are effective for all the primary and secondary members considered for analysis.

The total hull weight $(W)$, the weight of longitudinal members $\left(W_{L}\right)$ and the weight of transverse members $\left(W_{T}\right)$ of one hold length are plotted against the number of frame spaces in Fig. 9. It is clear from this figure that, as $N$ increases, the hull weight increases. The weight of transverse members also increases with increase in $N$, as the number of transverse frames increases. However, the weight of longitudinal members slightly decreases as $N$ increases the reason for which is as follows. The hull strength requirement is in excess in the case of plastic design. As $N$ increases the span length of the longitudinal members decreases and hence their scantlings (those which affect the hull strength) decrease so as to enable the local strength to get constrained. This results in the slight decrease in the weight of longitudinal members with increase in $N$.

\section{3 Comparison of Optimization Results Based on Plastic Design and Elastic Design}

In order to compare the proposed method with the optimization based on elastic design, optimization is carried out on the same standard ship based on elastic design using the SUMT method. The constraints considered for this analysis are as follows.

1) The maximum shear stress in a member should not exceed the allowable shear stress, subjected to a safety factor. This constraint is considered for the deck, side and bottom transverse members.

2 ) The maximum bending stress in a member should not exceed the allowable bending stress, subjected to a safety factor. This constraint is considered for the deck, side and bottom transverse members as well as for both the primary and secondary longitudinal members of deck and bottom.

3 ) The maximum bending stress in a local panel should not exceed the allowable stress, subjected to a safety factor. This constraint is considered for panels in bottom shell and deck plate.

4) The buckling stress in a local panel should not exceed the allowable buckling stress. The elastoplastic buckling constraint is considered for panels in bottom shell and deck plate.

5 ) The maximum bending stress and shear stress in a hull girder should not exceed the allowable bending stress and shear stress, respectively, subjected to a safety factor.

The above mentioned constraints in items 3 ) and 4)

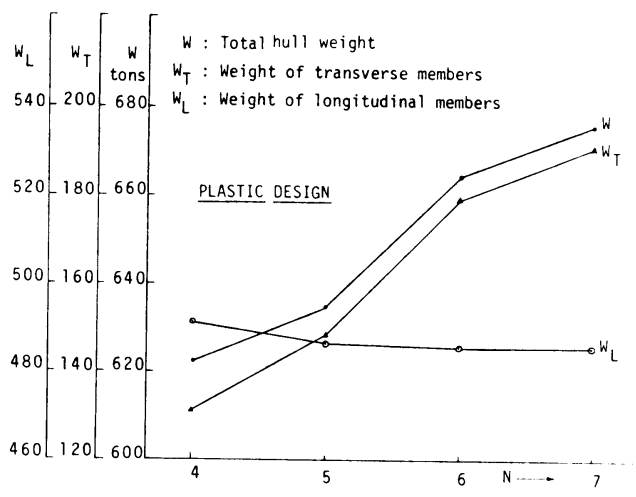

Fig. 9 Relationship between hull weight and number of frame spaces N (Plastic Design) 
are evaluated in the same way as that for plastic analysis. The maximum bending moments and shear forces are calculated by direct three dimensional structural analysis. The allowable stresses are based on NK Rule, which are shown in Table 6 . All other parameters and assumptions remain the same as that for the case of plastic design. The number of frame spaces is varied from 4 to 7 and for each of these cases $r_{1}$ is varied from $10^{3}$ to $10^{6}$ and the optimum design is chosen, as in the case of plastic design.

From the optimization results, it has been found that:

1) The shear stress constraint is effective for the deck, side and bottom transverses as well as for the hull girder.

2) The bending stress constraint is effective for the deck transverse and the hull girder.

Thus, the assumed constraints are effective for all the members considered for the analysis.

The total hull weight and the weights of longitudinal and transverse members of one hold length are plotted against the number of frame spaces in Fig. 10. It is clear from this figure that, as $N$ increases, the hull weight increases. The weight of transverse members also increases as in the case of plastic design. However, the weight of longitudinal members remains almost the same the reason for which is as follows. Since the hull girder scantling requirement is more in the case of elastic design, the hull strength depends on the scantlings of the longitudinal members. As $N$ increases, the

Table 6. Allowable stresses (Elastic design)

\begin{tabular}{|l|c|c|}
\hline \multicolumn{1}{|c|}{$\begin{array}{c}\text { Structural } \\
\text { Member }\end{array}$} & $\begin{array}{c}\text { Bending } \\
\text { Stress } \\
\left(\mathrm{kgf} / \mathrm{mm}^{2}\right)\end{array}$ & $\begin{array}{c}\text { Shear } \\
\text { Stress } \\
\left(\mathrm{kgf} / \mathrm{mm}^{2}\right)\end{array}$ \\
\hline Transverses & 18.0 & 8.5 \\
Longl. Girders & 10.0 & - \\
Secondary Longls. & 10.0 & - \\
Deck and Btm. plates & 20.0 & - \\
Hull & 20.0 & 10.0 \\
\hline
\end{tabular}

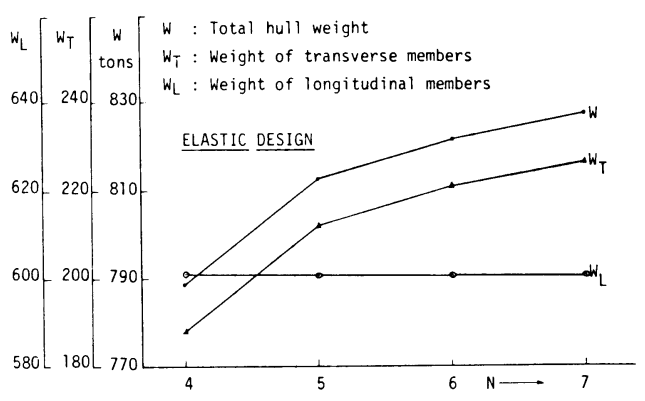

Fig. 10 Relationship between hull weight and number of frame spaces N (Elastic Design) local strength requirement of longitudinal members is in excess as their span length decreases. However, the scantlings of the longitudinal members cannot get reduced because of the constraint in hull strength as the hull strength depends on them. This keeps the weight of longitudinal members almost the same with increase in $N$.

It is worth noticing that the optimization results of both plastic and elastic designs are based on the respective design conditions assumed for the analyses. As these design conditions change, the optimization results may also change.

In the case of elastic design, the transverses and longi tudinal girders are affected by the constraints in bending stresses and shear stresses considered separately. On the other hand, in the case of plastic design, these members are affected by the constraints due to the fully plastic interactions between bending moments and shear forces. In the optimization of ship structures, it is reasonable to take the fully plastic interaction relationships (plastic design) rather than considering the bending moments and shear forces separately (elastic design), as the latter case may result in an overestimate of the design scantlings.

\section{Conclusions}

A procedure for the optimization of ship structures based on plastic design is developed using the Sequential Unconstrained Minimization Technique (SUMT) method. This procedure is applied in the optimization of a hold structure of a segregated ballast tanker taking one hold length bounded by two transverse bulkheads for analysis. Hull weight is taken as the objective function to be minimized.

The number of frame spaces $N$ in a hold length is five for the standard ship. Optimization is carried out for $N$ $=5$ considering various cases by varying the lower limit on variables $\boldsymbol{X}_{\min }$ from $90 \%$ to $50 \%$ of the initial values and the coefficient $r_{1}$ from $10^{3}$ to $10^{6}$, one at a time. The effect of $N$ on the hull weight is computed by varying $N$ from 4 to 7 . Since the hold length is kept as a constant, the transverse frame spacing varies with variation in $N$. A comparison of the proposed method with the optimization based on elastic design is also made. The results were analyzed and the following conclusions were arrived at.

(1) The proposed method is effective in the optimization of ship structures as is confirmed taking the case of the optimization of a hold structure of a segregated ballast tanker. The hull weight of the hold length analyzed has got reduced.

(2) In order to reduce the computation time for structural analyses in the optimization process, the variations of maximum bending moments and shear forces in structural members due to change in design variables are calculated using Taylor series expansion method. In this case, a 
lower limit has to be set on the design variables in order to effectively optimize a ship structure using the proposed method. In the present analyses the lower limit is set at $70 \%$ of the respective initial values of the initial ship so that the changes in moments are within the allowable limit.

(3) The proposed method using the Taylor series expansion has resulted in a saving of $57 \%$ in CPU time compared to the direct method.

(4) As the number of frame spaces increases, the total hull weight and the weight of transverse members increases. The weight of longitudinal members slightly decreases in the case of plastic design and it remains almost the same in the case of elastic design.

(5) In setting up the constraints, the bending moments and shear forces considered separately (in the case of elastic design) may result in an overestimate of the design variables, and therefore it is more reasonable to consider their fully plastic interaction relationships (in the case of plastic design), in the optimization of ship structures.

The numerical computations in this paper were carried out using HITAC $M-680 \mathrm{H}$ of the Information Processing Center of Hiroshima University.

\section{References}

1) Moe, J. and Lund, S., "Cost and Weight Minimization of Structures with Special Emphasis on Longitudinal Strength Members of Tankers," Trans. RINA, Vol. 110, 1968, pp. 43-70.

2) Kitamura, K., "Studies on Optimization of Ship Structures (2nd Report)," J. Soc. Naval Arch. of Japan, Vol. 130, 1971, pp. 259-268 (in Japanese).

3) Higuchi, M., "Optimum Design of Indefinitely Long Plate with Reinforcements," J. Soc. Naval Arch. of Japan, Vol. 131, 1972, pp. 319-327 (in Japanese).

4) Nobukawa, H., "On the Optimum Design of Ship Structures in consideration of the Three-Dimensional Strength ( I )," Trans. West Japan Soc. of Naval Arch., Vol.46, 1973, pp. 129-141 (in Japanese).

5) Ryu, Y.S., Harrison, M., Wu, C.C. and Arora, J. S., "Structural Design Sensitivity Analysis of Nonlinear Response," Comput. Struct., Vol. 21, No. 1/2, 1985, pp. 245-255.

6) Haftka, R. T. and Mroz, Z., "First and Second Order Sensitivity Analysis of Linear and Nonlinear Structures," AIAA J., Vol. 24, No. 7, 1986, pp. 1187-1192.

7) Wu, C. C. and Arora, J. S., "Design Sensitivity Analysis and Optimization of Nonlinear Structural Response Using Incremental Procedure," AIAA J., Vol. 25, No. 8, 1987, pp. 1118-1125.

8) Yao, T., Abdul Rahim, M. and Kato, T., "Sensitivity Analysis and Reanalysis of Elastoplastic Plane Stress Problems," J., Soc. Naval Arch. of Japan, Vol. 166, 1989, pp. 277-284.

9) Yamaguchi, I., "Approximate Method on the
Calculation on Transverse Strength of Ship (Second Report)," J., Soc. Naval Arch. of Japan, Vol. 109, 1961, pp. 213-227 (in Japanese).

10) Nippon Kaiji Kyokai, Rules and Regulations for the Construction and Classification of Ships, Amendment, 1976.

11) Kusuda, T., "Plastic Analysis of Plate Structures Subjected to Transverse Load," J., Soc. Naval Arch. of Japan, Vol. 107, 1960, pp. 195-202 (in Japanese).

\section{Appendix I : Optimization Flow Chart}

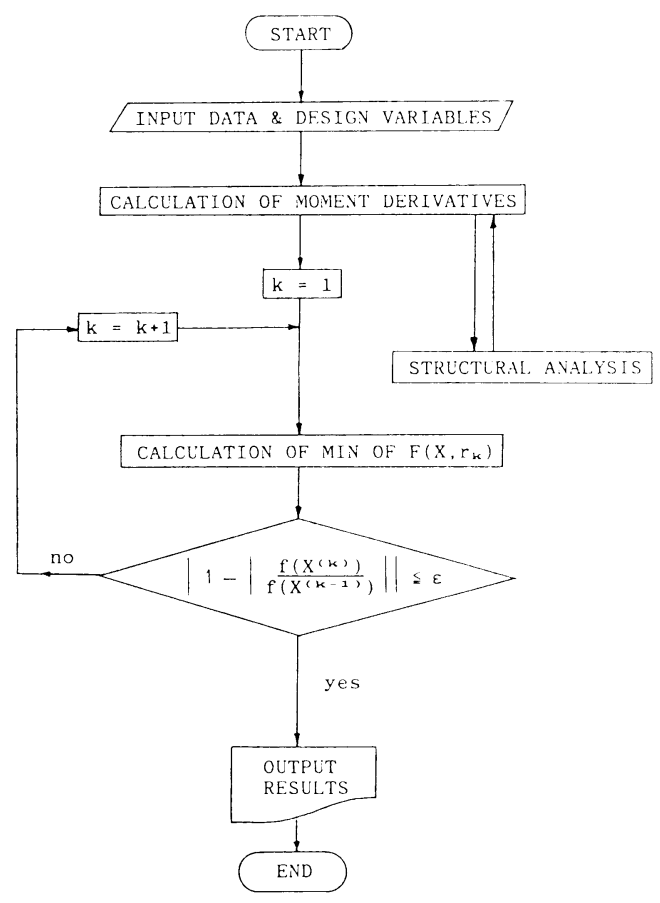

Appendix II

The state vectors at frame point 1 to the left $\boldsymbol{V}_{1}^{l}$ and at frame point $n$ to the right $\boldsymbol{V}_{n}^{R}$ are

$$
\boldsymbol{V}_{\mathrm{I}}=\boldsymbol{V}_{n}^{R}=\left[\begin{array}{l}
w_{B}=0 \\
\phi_{B}=0 \\
Q_{B} \\
M_{B} \\
w_{D}=0 \\
\phi_{D}=0 \\
Q_{D} \\
M_{D} \\
1
\end{array}\right]
$$

Subscripts ${ }_{B}$ and ${ }_{b}$ represents bottom and deck respectively.

The initial matrix, $\quad \boldsymbol{A}=\left[\begin{array}{l}Q_{B} \\ M_{B} \\ Q_{D} \\ M_{D} \\ 1\end{array}\right]_{1}$ 
The aft end matrix, $\quad \boldsymbol{R}_{L}=\left[\begin{array}{ccccc}0 & 0 & 0 & 0 & 0 \\ 0 & 0 & 0 & 0 & 0 \\ 1 & 0 & 0 & 0 & 0 \\ 0 & 1 & 0 & 0 & 0 \\ 0 & 0 & 0 & 0 & 0 \\ 0 & 0 & 0 & 0 & 0 \\ 0 & 0 & 1 & 0 & 0 \\ 0 & 0 & 0 & 1 & 0 \\ 0 & 0 & 0 & 0 & 1\end{array}\right]$

The forward end matrix,

$\boldsymbol{R}_{R}=\left[\begin{array}{lllllllll}1 & 0 & 0 & 0 & 0 & 0 & 0 & 0 & 0 \\ 0 & 1 & 0 & 0 & 0 & 0 & 0 & 0 & 0 \\ 0 & 0 & 0 & 0 & 1 & 0 & 0 & 0 & 0 \\ 0 & 0 & 0 & 0 & 0 & 1 & 0 & 0 & 0\end{array}\right]$

The field matrix of the segment $i$,

$$
\boldsymbol{F}_{i}=\left[\begin{array}{ccccccccc}
1 & a+l+b & F 1 & F 2 & 0 & 0 & 0 & 0 & 0 \\
0 & 1 & F 3 & F 4 & 0 & 0 & 0 & 0 & 0 \\
0 & 0 & 1 & 0 & 0 & 0 & 0 & 0 & 0 \\
0 & 0 & a+l+b & 1 & 0 & 0 & 0 & 0 & 0 \\
0 & 0 & 0 & 0 & 1 & a+l+b & F 5 & F 6 & 0 \\
0 & 0 & 0 & 0 & 0 & 1 & F 7 & F 8 & 0 \\
0 & 0 & 0 & 0 & 0 & 0 & 1 & 0 & 0 \\
0 & 0 & 0 & 0 & 0 & 0 & a+l+b & 1 & 0 \\
0 & 0 & 0 & 0 & 0 & 0 & 0 & 0 & 1
\end{array}\right]
$$

where

$$
\begin{aligned}
& F 1=(-l / E I)\left[l a / 2+l^{2} / 6+a b+l b / 2\right]+l /(G A) \\
& F 2=(-l / E I)[b+l / 2] \\
& F 3=(-l / E I)[a+l / 2] \\
& F 4=-l / E I
\end{aligned}
$$

$F 1, F 2, F 3$ and $F 4$ are for the bottom member and $F 5$, $F 6, F 7$ and $F 8$ are similar values for the deck member. The point matrix at the frame point $i$,

$$
\boldsymbol{P}_{i}=\left[\begin{array}{ccccccccc}
1 & 0 & 0 & 0 & 0 & 0 & 0 & 0 & 0 \\
0 & 1 & 0 & 0 & 0 & 0 & 0 & 0 & 0 \\
-R_{B}^{B} & 0 & 1 & 0 & -R_{B}^{D} & 0 & 0 & 0 & -R_{B} \\
0 & 0 & 0 & 1 & 0 & 0 & 0 & 0 & 0 \\
0 & 0 & 0 & 0 & 1 & 0 & 0 & 0 & 0 \\
0 & 0 & 0 & 0 & 0 & 1 & 0 & 0 & 0 \\
-R_{D}^{B} & 0 & 0 & 0 & -R_{D}^{D} & 0 & 1 & 0 & R_{D} \\
0 & 0 & 0 & 0 & 0 & 0 & 0 & 1 & 0 \\
0 & 0 & 0 & 0 & 0 & 0 & 0 & 0 & 1
\end{array}\right]
$$

where $R_{B}$ and $R_{D}$ are the vertical reaction forces acting on bottom and deck center girders respectively at frame point $i$ under full load condition. $R_{B}^{B}$ and $R_{D}^{B}$ are similar values with unit deflection at bottom girder. Similarly, $R_{B}^{D}$ and $R_{D}^{D}$ are with unit deflection at deck girder. 